Trauma Berufskrankh 2004 - 6 [Suppl 4] : S396-S407 DOI 10.1007/s10039-004-0844-2

Online publiziert: 29. April 2004

(c) Springer-Verlag 2004
D.-H.Boack

Sektion Fuß- \& Sprunggelenkchirurgie, Klinik für Unfall- und Wiederherstellungschirurgie,

Charité Campus Virchow, Berlin

\section{Fraktur des oberen Sprunggelenks}

\section{Therapeutische Strategien}

N ach klinischer und bildgebender Diagnostik müssen die therapierelevanten Entscheidungen getroffen werden. Dazu sind klare strategische Behandlungsziele und ein individuelles Versorgungskonzept erforderlich.

Der Sprunggelenkbruch ist eine Luxationsfraktur mit knöchernem, kapsuloligamentärem und kartilaginärem Verletzungsmuster. Es weist sehr unterschiedliche Weichteilschädigungen auf. Es ist daher notwendig, die Weichteilrestitution mit in das Gesamtkonzept einzubeziehen. In der Behandlungstaktik müssen deswegen oft die Akutbehandlung und die definitive Therapie voneinander getrennt werden.

Strategisches Ziel sind die anatomische Gelenkreposition aller beteiligten Facetten in sich und die Wiederherstellung der anatomischen Position der Facetten zueinander. Dafür sind die Rekonstruktion der Achse, der Länge und der Rotation der Fibula und die Protektion der ligamentären Konsolidierung unerlässlich. Eine stabile Retention ist für eine adäquate Nachbehandlung obligat. Nur so können eine zeitgerechte knöcherne und ligamentäre Heilung erreicht und sowohl Stabilität als auch Beweglichkeit erzielt werden.

\section{Notfallbehandlung}

\section{Unfallort}

Nach der ersten Blickdiagnostik, bei der offene Frakturen, persistierende Luxatio- nen und ein seltenes Kompartmentsyndrom erkannt werden müssen, wird entsprechend der akuten Analyse eine Notfallbehandlung eingeleitet. Sie beinhaltet die Notfallreposition, ggf. mit temporärer Transfixation, und eine konsequente Weichteiltherapie (• Abb. 1).

Entsprechend der vorwiegend indirekten Krafteinwirkung - in fast 90\% liegt ein „Distorsionstrauma“ vor - wird die Weichteilschädigung maßgeblich durch den Fragmentdruck von innen, d. h. die Luxationsstellung, bestimmt. Insbesondere die anteromedialen Weichteile können durch den scharfkantigen Innenknöchelbruchrand von innen kompromittiert werden. Daraus muss als therapeutische Taktik eine sofortige Reposition jeglicher Luxationsstellungen, auch bei (noch) geringem Weichteilschaden, gefordert werden. Diese notfallmäßige geschlossene Reposition und Retention in einer geeigneten Schiene sind sofort, noch am Unfallort bzw. durch den erstuntersuchenden Arzt, vorzunehmen.

\section{Klinik}

Nach der Übergabe durch den einliefernden Notarzt bzw. Rettungssanitäter sowie einer Exploration und erneuten klinischen (Ganzkörper-)Untersuchung am entkleideten Patienten in der Rettungsstelle des versorgenden Krankenhauses wird geröngt. Falls klinisch eine Fehlstellung im- poniert, sind die Röntgenaufnahmen sofort in Arztbegleitung als Notfalluntersuchung durchzuführen. Anschließend erfolgen ggf. die sofortige Nachreposition in Analgesie und Gipsretention.

Für die Reposition liegt der Patient entspannt in Rückenlage, mit gebeugtem Hüft- und Kniegelenk. Sie beginnt mit einem allmählichen lokalen Händedruck zum „Wegdrücken“ der Schwellung und Längszug an der Ferse, falls erforderlich in Lokalanästhesie (Gelenkpunktion medial der Tibialis-anterior-Sehne). Geringe weitere Korrekturen zum Ausgleich der häufigen lateralen und dorsalen Talusverschiebung mit Außenrotationsfehlstellung werden durch Druck und Gegendruck über die 2. Hand an der distalen Tibia („Stiefelausziehen“) am sichersten unter Bildwandlerkontrolle realisiert (- Abb. 2).

Das Anlegen und Aushärten des nur lokal gepolsterten Gipses (s. u.) erfolgen unter permanentem Zug erst nach der Einrichtung. Die erreichte Stellung des OSG wird im Gips radiologisch in 2 Ebenen dokumentiert.

\section{Weitere Entscheidungsfindung und Primärtherapie}

Für die weitere Entscheidungsfindung sind eine subtile Weichteil- und Röntgenanalyse und die Bewertung mit nachfolgender Klassifikation von Fraktur und Weichteilschaden erforderlich. Insbeson- 
dere der Weichteilschaden und der Grad der Instabilität bestimmen das Vorgehen am Unfall- bzw. Einlieferungstag.

\section{Fixateur externe}

Bei jeglicher Redislokation im Gips, allen hochgradig instabilen Situationen und bei G3-Situationen, lokalen Hautschäden bzw. zweit- und drittgradig offenen Frakturen oder Fragmentdruck von innen sollte $u$. a. eine dringliche Transfixation mit einem Fixateur externe erfolgen. Auch bei polytraumatisierten Patienten ist eine semioperative Primärtherapie mit einem Fixateur externe zur Sprunggelenktransfixation sinnvoll, da damit eine sichere Retention sowie eine gute Weichteilbehandlung und -beurteilung möglich sind. Meist ist eine tibiometatarsale Transfixation mit einem monolateralen Fixateur ausreichend. Allerdings sollte nach der Transfixation immer eine Stabilitätskontrolle im OP durchgeführt werden. Bei hochgradig instabilen trimalleolaren OSG-Luxationsfrakturen (B3- und C-Frakturen) ist eine zusätzliche Rückfußtransfixation mittels Kalkaneus-SteinmannNagel mit mittigem Gewindeanteil erforderlich. Hierbei ist auch auf die erforderliche stabile Dreieck-Rahmen-Konstruktion zu achten. Ansonsten kann es selbst im Fixateur zur Redislokation kommen (• Abb. 3).

Der Eingriff wird in Allgemeinnarkose in Rückenlage mit intraoperativer Bildwandlerkontrolle durchgeführt. In seltenen Fällen kann der Fixateur auch nach der definitiven Osteosynthese zur Weichteilbehandlung weiter verwendet werden, wenn eine gipsfreie Nachbehandlung aus Weichteilgründen nicht möglich ist.

\section{Alternativmethoden}

Früher verwendete Alternativmethoden mit einer Fersenbeindrahtextension, die eine völlige Immobilisierung des Patienten notwendig macht, sollten der Vergangenheit angehören.

Nur bei lagerungsstabilen Frakturen und blanden Weichteilverhältnissen kann die primäre Ruhigstellung im Unterschenkelspaltgipsverband bis zur definitiven Therapie fortgeführt werden.

Trauma Berufskrankh 2004 - 6 [Suppl 4] : S396-S407

DOI 10.1007/s10039-004-0844-2

C) Springer-Verlag 2004

\section{D.-H. Boack}

\section{Fraktur des oberen Sprunggelenks. Therapeutische Strategien}

\section{Zusammenfassung}

Für ein optimales Behandlungsergebnis bei Frakturen des oberen Sprunggelenks ist ein abgestuftes Therapieschema erforderlich, das den Weichteilschaden und den Frakturtyp bzw. den Schweregrad der Instabilität, aber auch bestehende Erkrankungen in die Behandlungstaktik einbezieht. Unabhängig von der definitiven Therapieform müssen starke Dislokationen oder Luxationen durch eine präliminare Reposition als notfallmäßige Sofortmaßnahme und geeignete Retention am Unfallort behandelt werden. Die anatomische Rekonstruktion der beteiligten Ge- lenkflächen, die Wiederherstellung der normalen Stellung aller Gelenkpartner zueinander, die Refixation oder Entfernung osteochondraler Fragmente und die Reparatur des Bandapparats mit einem möglichst übungsstabilen Verfahren stellen die Methode der Wahl dar. Der Weichteilschaden determiniert jedoch das Timing entscheidend und beeinflusst die Verfahrenswahl wesentlich.

\section{Schlüsselwörter}

OSG-Luxationsfrakturen · Therapie · Konzept

\section{Fracture of the ankle. Treatment strategies}

\section{Abstract}

For an optimal result to be achieved after a displaced ankle fracture it is essential for a graduated treatment schedule taking account not only of the soft tissue damage and the fracture type and/or severity of the instability, but also of any pre-existing illnesses to be set up. Whatever form the definitive therapy is to take, any severe dislocation should be reduced as an emergency procedure at the site to protect the soft tissues. All joint facets must be reduced anatomically, which means that open reduction plus internal fixation is the treatment of choice in almost all cases. However, the timing of the operative treat- ment and the options open for surgery are determined by the nature and severity of the soft tissue lesions. Conventional standard X-rays are sufficient to classify most ankle fractures. Anatomical reduction and stable internal fixation together with adequate physical therapy are apparently essential for, but do not definitively guarantee, a good result.

\section{Keywords}

Ankle fracture · Treatment options · Treatment schedule 


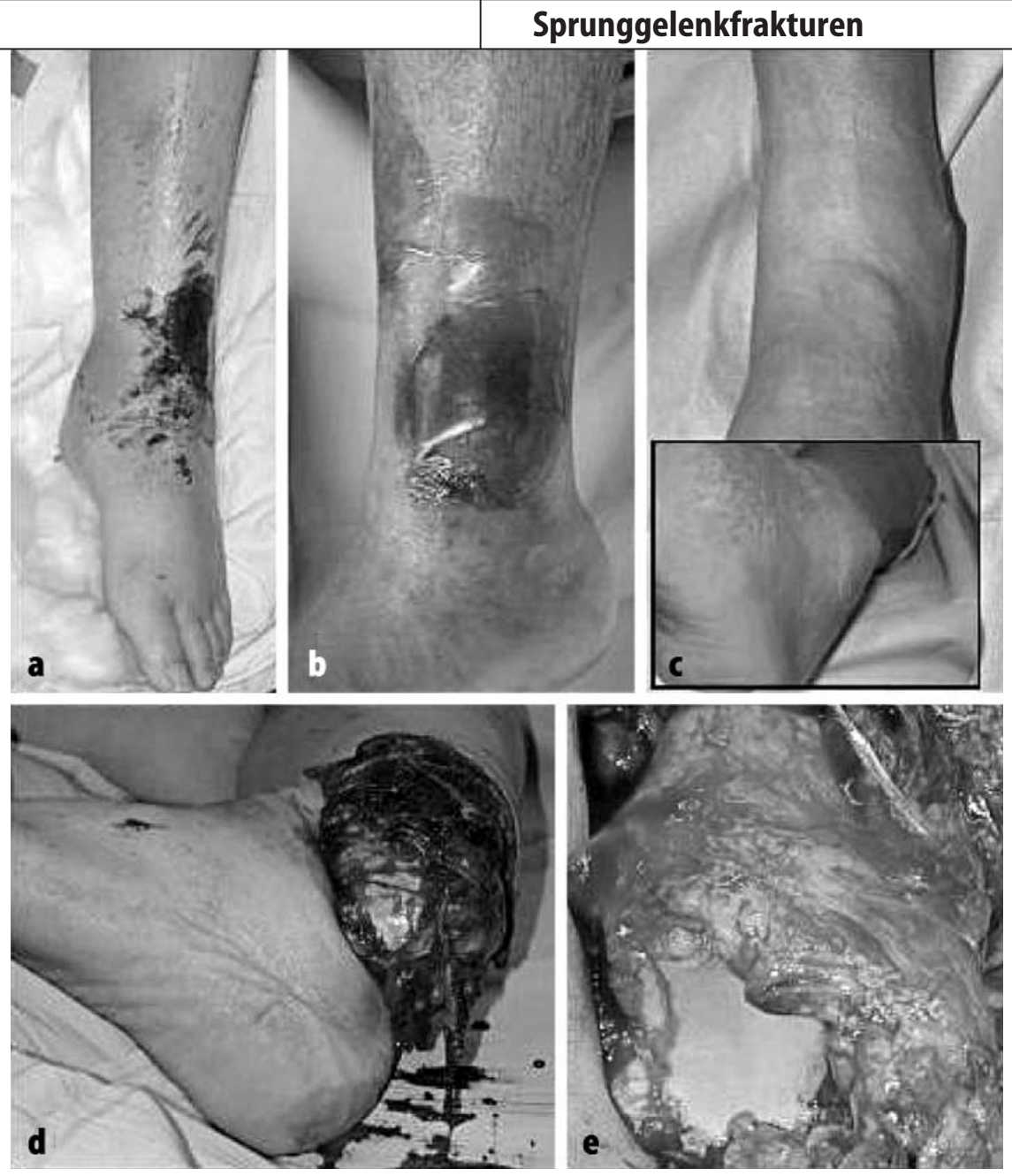

Abb. 1a-e $\Delta$ Weichteilschäden bei OSG-Luxationsfrakturen, (a) zweitgradig geschlossener Weichteilschaden mit alten verschorften Exkoriationen nach Zuverlegung aus dem Ausland bei unversorgter Fraktur; (b) Spannungsblasen bei drohendem Kompartmentsyndrom (G3); (c) frische Fraktur mit globalem Weichteilschaden G1, aber lokalem Fragmentdruck von innen und drohender, erstgradig offener Fraktur; (d) 3A-offene OSG-Luxationsfraktur (A3-Adduktionsfraktur) vor und (e) nach Débridement
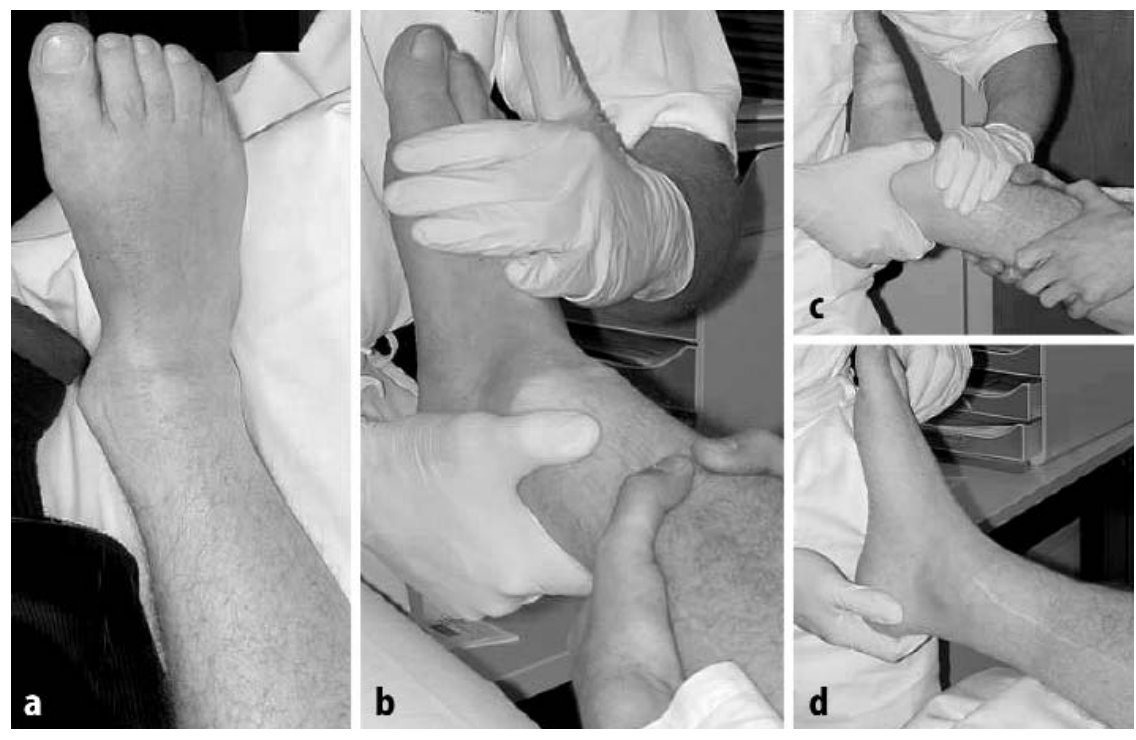

Abb. 2a- $d \boldsymbol{\Delta}$ Notfallreposition am Beispiel einer meist bestehenden posterolateralen (Sub-)Luxation (a) mit schonender Reposition durch Zug- und Gegendruck (b, c) und reponiertem Gelenk (d)

\section{Diagnostik}

Die Zeit bis zur endgültigen Therapie wird für das Weichteilmanagement und ggf. für eine erweiterte bildgebende Diagnostik genutzt. Nur bei blanden lokalen Verhältnissen können diese ambulant durchgeführt werden. Neben der regelmäßigen Reevaluierung der Weichteile sind eine nochmalige sorgfältige anamnestische Erhebung und klinische Untersuchung erforderlich (Diabetes mellitus, Polyneuropathie, PAVK, Immundefizienz usw.). Insbesondere bei polytraumatisierten oder polymorbiden Patienten muss die Lagerung mehrfach täglich kontrolliert werden. In Abhängigkeit von diesen Untersuchungen wird der Behandlungsplan mit zeitlicher Abfolge und Behandlungsmethode aufgestellt.

Nach der Klassifikation des Weichteilschadens wird meist die AO-Klassifikation verwendet, um die Indikation zum therapeutischen Vorgehen abzuleiten. Die pathogenetische Einteilung nach LaugeHansen kann für die „Suche“ nach zunächst okkulten Zusatzfrakturen und für das Repositionsmanöver bzw. in einigen Fällen für die Therapiewahl hilfreich sein.

\section{Therapiestrategie}

Unabhängig von der definitiven Therapieform müssen starke Dislokationen oder Luxationen durch eine präliminare Reposition als notfallmäßige Sofortmaßnahme und geeignete Retention am Unfallort behandelt werden.

Jede OSG-Fraktur wird, wenn sie aus Weichteilgründen (6-h-Grenze) nicht sofort osteosynthetisch versorgt werden kann, primär so therapiert, dass sie auch in dieser Form ausbehandelt werden könnte.

Die anatomische Rekonstruktion der beteiligten Gelenkflächen, die Wiederherstellung der normalen Stellung aller Gelenkpartner zueinander, die Refixation oder Entfernung osteochondraler Fragmente und die Reparatur des Bandapparats mit einem möglichst übungsstabilen Verfahren bilden die Methode der Wahl. 


\section{Konservative Therapie}

Sie ist nur dann indiziert, wenn eine anatomische Situation besteht oder geschlossen geschaffen und eine sichere Retention garantiert werden können. Zur konservativen Behandlung eignen sich

- nicht oder minimal dislozierte infrasyndesmale Fibulafrakturen (A1-Frakturen)

- isolierte unverschobene Innenknöchelbrüche (A2.1-Frakturen)

- unverschobene bzw. gering verschobene isolierte B1.1-Frakturen bei geringem Weichteilschaden

Weiterhin können lokale oder selten allgemeine Kontraindikationen bzw. der Patientenwunsch zu einer nicht operativen Behandlung zwingen.

Initial wird meist in einem Unterschenkelspaltgipsverband bzw. Cast ruhig gestellt. Nach dem Abschwellen kann mit einer stabilen OSG-Orthese oder auch funktionell nachbehandelt werden - vorausgesetzt, die initiale Stressdiagnostik hat eine OSG-Stabilität erbracht. Die Entscheidung sollte u. a. vom Weichteilbefund und der Patientencompliance abhängig gemacht werden. Zunehmend wird eine symptomadaptierte Belastung empfohlen. Ob sich bei diesen stabilen Frakturen mit einer primär funktionellen Therapie langfristig gleich gute Ergebnisse erzielen lassen, wird derzeit in klinischen Studien evaluiert.

Falls eine Ruhigstellung erforderlich ist, müssen folgende Details der Gipsbehandlung berücksichtig werden: Der Patient wird rechtzeitig über das erforderliche Prozedere (Hochlagerung, Eis und mögliche neurovaskuläre Komplikationen und deren Prophylaxe) aufgeklärt. Der Unterschenkelspaltgipsverband wird in entspannter liegender oder sitzender Patientenposition angebracht. Der Spaltschlauch wird angelegt, wobei auf eine gute Polsterung von Fibulaköpfchen, Tibiavorderkante, Malleolen, Ferse und Metatarsale-V-Basis geachtet wird. Der Gips schließt lateral das Fibulaköpfchen (Peronäusprotektion), ventral die Tuberositas tibiae (dorsal aber freie Kniebeugung) und distal die Metatarsalia (Fußrückenödem) ein, während distal alle Zehen dorsal frei bleiben. Das OSG muss von

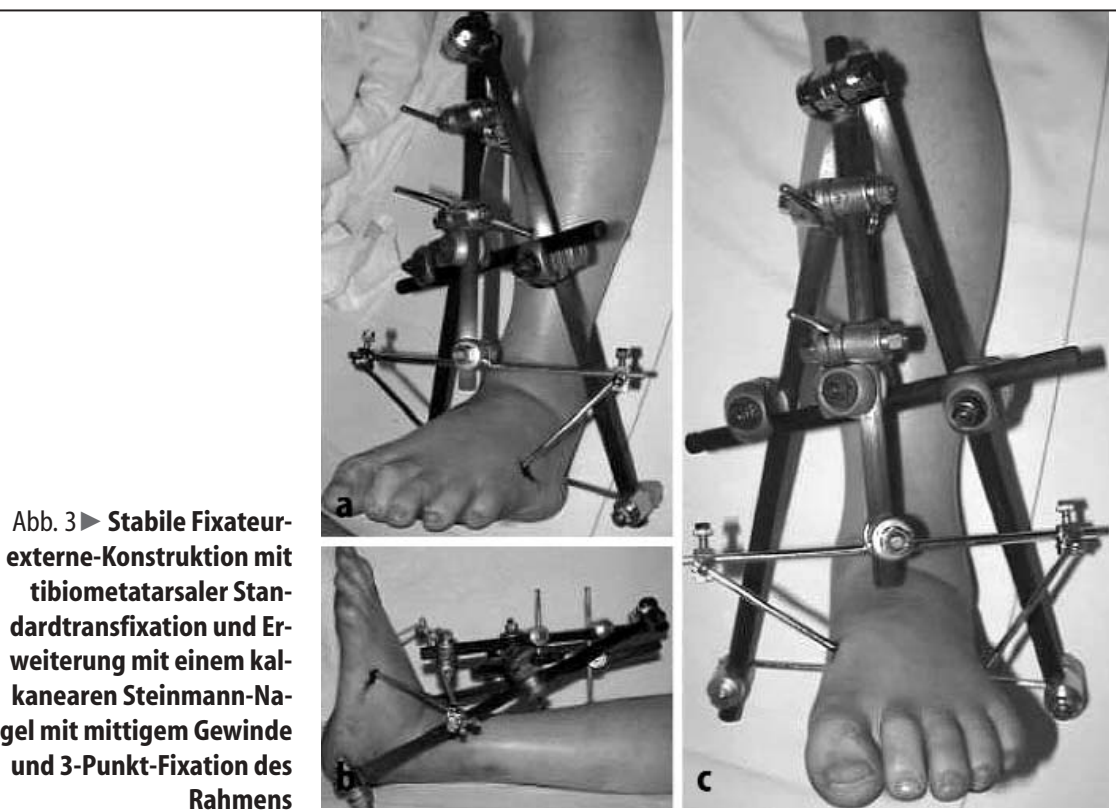

Beginn an in Neutralstellung gehalten werden, insbesondere sind Spitzfuß- oder Supinationsstellungen $\mathrm{zu}$ vermeiden.

Die Gipsbinden werden abgewickelt, nicht gezogen, um Schnürfurchen zu vermeiden. Jede Bindentour bedeckt die vorhergehende zu zwei Drittel. Die Sohlenplatte wird sorgfältig modelliert. Bei härtendem Gips kann keine Stellungskorrektur mehr erfolgen, da sich dabei Schnürfurchen am Rist bilden. Das Aushärten erfolgt auf einer elastischen Unterlage (Achillessehnenrolle). Die Spaltung muss vollständig bis zur letzten Faser durchgeführt werden. Zur Vermeidung von Druckstellen werden die Ränder am Schluss mit dem Finger „unterfahren".

Nachfolgend werden eine Röntgenkontrolle im Gips durchgeführt und der Gips beschriftet (Bruchtyp/Datum/Signum). Alternativ kann auch primär ein Cast verwendet werden.

Die Thromboseprophylaxe beginnt mit der Erstapplikation am Unfalltag. Unterarmgehstützen werden ausgehändigt und je nach Stabilität eine Teilbelastung von $15 \mathrm{kp}$ empfohlen. Eventuell ist die Gabe von antiphlogistisch-analgetisch wirksamen Pharmaka zu erwägen.

Der Gips wird spätestens nach $24 \mathrm{~h}$ kontrolliert und nach Weichteilkonsolidierung mit Röntgenkontrolle nach etwa 1 Woche, ggf. unter Repositionsbedingungen, mit Absatz und Sohlenanlage gewechselt, evtl. wird sekundär ein Cast ap- pliziert. Bei stabilen Frakturen kann eine symptomadaptierte Vollbelastung im Cast durchgeführt werden.

Die Dauer der Immobilisation beträgt etwa 6 Wochen. Nach Gipsabnahme werden eine aktive Krankengymnastik und Stützstrümpfe für weitere 4 Wochen verordnet.

\section{Operative Therapie}

\section{Indikation}

Offene Reposition und interne Fixation sind indiziert bei:

- geschlossen nicht reponierbaren bzw. nicht retinierbaren dislozierten A1Frakturen;

- dislozierten, instabilen A2-/A3-Adduktionsfrakturen;

- dislozierten und nicht reponierbaren B1.1-Frakturen, da eine unverkürzte Fibula ohne Rotationsfehler und laterale Dislokation nur operativ hergestellt werden kann;

- alle weiteren B- (ab B1.2-Fraktur aufwärts) und C-Frakturen, da geschlossen fast nie ein exakter Gabelschluss und eine anatomische Fragmentsituation geschaffen werden können;

Unabhängig davon müssen alle offenen Frakturen operativ angegangen werden. Auch selten vorliegende Kompartmentsyndrome zwingen zur akuten Dekompression. 

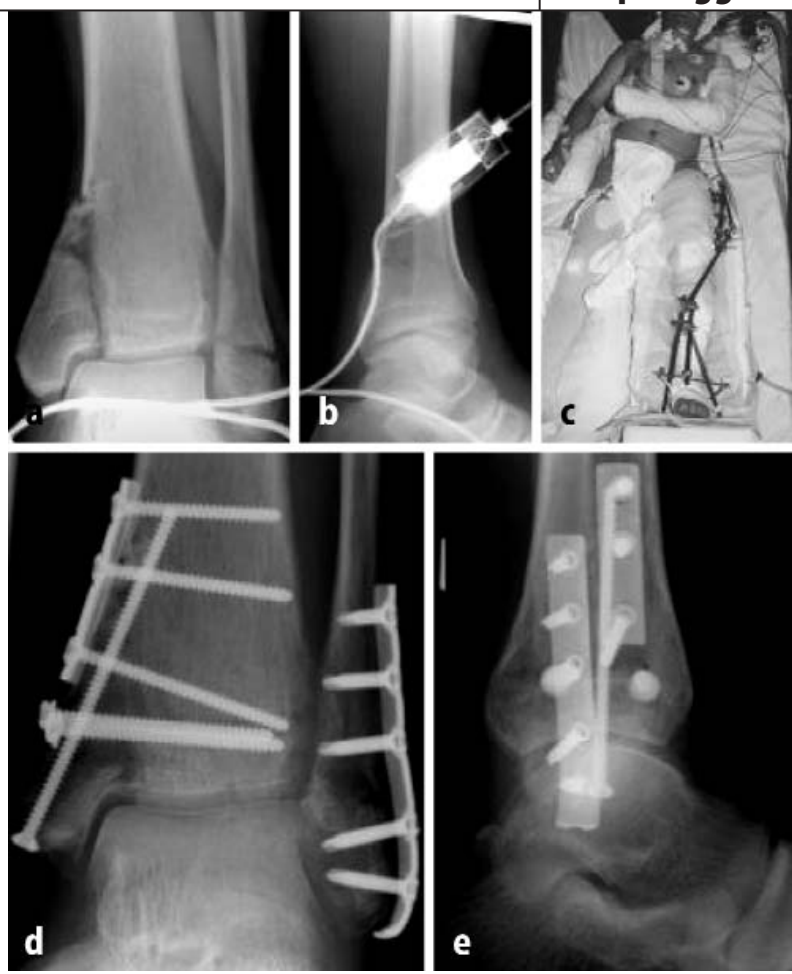

Abb. 4a-e $<$ Unfallaufnahmen nach Notfallreposition der Luxation mit infrasyndesmaler querer Fibulaabrissfraktur und medialer Adduktionsabscherimpressionskomponente $(a, b)$; aufgrund der Instabilität und des eichteilschadens bei polytraumatisierten Patienten: primäre Transfixation der Kettenverletzung der linken unteren Extremität (c) und postprimäre Versorgung mit Platten-Schrauben-0steosynthese $(d, e)$

Weitere therapierelevante Begleitverletzungen sind osteochondrale Abscherfrakturen meist von der anterolateralen Talusrolle sowie Sehnenluxationen der Peronäal- und seltener der Tibialis-posterior-Sehne.

\section{Operationsplanung}

Der beste Zeitpunkt für die Osteosynthese liegt sofort innerhalb der 6-h-Grenze, falls die Weichteilsituation dies zulässt oder wenn sie es erfordert (offene Frakturen, Kompartmentsyndrom, Fragmentdruck von innen). Sonst werden primär eine Gipsretention oder eine temporäre Transfixation mit nachfolgender konsequenter Hochlagerung, Kryotherapie ggf. mit AV-Impulsgenerator und medikamentöser Antiphlogese durchgeführt. Die endgültige Therapie erfolgt in diesen Fällen nach Weichteilkonsolidierung.

Eine präoperative Zeichnung ist eine gute Planungsgrundlage, um die operative Taktik festzulegen und vor dem Eingriff das Vorhandensein der notwendigen Implantate und deren Größe ggf. zu überprüfen.

\section{Lagerung}

Bei isolierten Außenknöchelfrakturen oder bei bimalleolaren Brüchen empfiehlt sich als Standard die Rückenlage mit Keil- kissen unter der ipsilateralen Gesäßhälfte und unterpolstertem Unterschenkel mit kontralateral abgesenkter unterer Extremität, sodass bequem ein epimalleolarer Zugang gewählt und eine intraoperative Röntgenkontrolle gewährleistet werden können. Für die direkte Stabilisierung des Volkmann-Fragments über einen posterolateralen Zugang ist die Bauchlage geeignet, wobei auch die Knöchel versorgt werden können.

\section{Zugänge}

Nach dem sterilen Anstrich des gesamten Unterschenkels werden die knöchernen „Landmarken“ und die Schnittführung eingezeichnet. Längsschnitte sind vorzuziehen, da sie die Lymphgefäße schonen und problemlos verlängerbar sind. Der Standardzugang verläuft epimalleolar über dem frakturierten Knöchel. Falls erforderlich, kann distal der Malleolarspitze die Inzision leicht bogenförmig nach ventral-distal erweitert werden, um eine ventrale Präparation und vordere Gelenkdarstellung zu erleichtern. Die Inzision erfolgt mit dem Skalpell in einem Schnitt bis auf das Periost. Weichteilschonendes Operieren bedeutet hier den Verzicht auf eine Blutsperre, auf brüsken oder stoischen Hakenzug und auf das übermäßige Mobilisieren des Weichteillappens. Die Fragmente werden epiperiostal nur so weit dargestellt, wie für die Reposition bzw. Retention erforderlich. Eine marginale Bruchflächenreinigung wird scharf durchgeführt, um eine anatomische Reposition zu ermöglichen.

Lateral wird bei der Schnittführung der evtl. atypische Verlauf des superfizialen Peronäusasts oder des N.-suralis-lateralis-Endasts über dem Außenknöchel beachtet.

Der mediale Zugang erfolgt ebenfalls als epimalleolare Längsinzision unter Schonung der ventral verlaufenden V. saphena magna bzw. des N.-saphenus-Endasts (cave: Neurombildung) und subtiler Ligierung von oft kräftigen Gefäßen des Rete malleolare. Durch das im Frakturverlauf rupturierte Periost können die Fraktur direkt dargestellt und zugleich das Gelenk inspiziert werden. Dorsal endet die häufig quere Fraktur im Postikussehnenlager, dessen synoviale Auskleidung sorgfältig geschont werden muss, um Sehnenadhäsionen zu vermeiden.

Für den posterolateralen Zugang wird die Hautinzision mittig zwischen Achillessehnenrand und Außenknöchelhinterkante vorgenommen und distal leicht bogenförmig um die Malleolusspitze nach ventral geführt. Nach Subkutisspaltung sollten die V. saphena parva und der N. suralis dargestellt und geschont werden. Danach wird die Unterschenkelfaszie über dem M. flexor hallucis longus gespalten. Dieser wird nach medial und die Peronäalsehnen nach lateral retrahiert, die Vasa peroneae posteriores werden geschont, ggf. gering mobilisiert, und die Fraktur wird dargestellt. Die Indikation für diesen Zugang besteht bei großen versorgungspflichtigen Volkmann-Fragmenten ohne intermediäre Gelenkimprimate, wobei die Fibulafraktur über diesen Zugang von dorsal versorgt werden kann.

Bei der kombinierten Innenknöchelund Volkmann-Fraktur, insbesondere beim Vorliegen von interponierten $\mathrm{Ge}$ lenkzwischenfragmenten, kann der modifizierte dorsomediale Zugang gewählt werden. Hier erfolgt die Schnittführung im Postikussehnenverlauf. Nach geringer subkutaner Präparation können in oben genannter Weise einerseits das Malleolarfragment dargestellt und andererseits 
nach Inzision der Postikussehnenscheide und Sehnenretraktion nach dorsal auch das Volkmann-Fragment eingesehen werden. Über diesen Zugang erfolgt die direkte Reposition sowohl des Volkmannals auch des Malleolarfragments mit direkter Verschraubung.

Der klassische posteromediale Zugang dagegen wird bei intaktem Innenknöchel gewählt, wenn die mehrfragmentäre Volkmann-Fraktur große interponierte $\mathrm{Ge}$ lenkzwischenfragmente aufweist und daher direkt unter Sicht reponiert werden muss. Hierbei wird eine schräge Inzision etwa einen Querfinger dorsal der Innenknöchelhinterkante geführt. Nach Hautund Subkutisinzision wird das obere Flexorenretinaculum dargestellt und eröffnet. Es erfolgt die subtile Darstellung des neurovaskulären Bündels en bloc, d. h. A. und Vv. tibiales posteriores und N. tibialis posterior werden mit dem begleitenden subkutanen Gewebe nach dorsal retrahiert, nachdem sie proximal und distal gelöst worden sind. Unmittelbar darunter kommen die OSG-Gelenkkapsel und die meist hier auslaufende mediale Fraktur des Volkmann-Fragments zur Darstellung. In Abhängigkeit vom Frakturverlauf bleibt die Postikussehnenscheide fast generell unberührt, und die M.-flexor-digitorum-longus-Sehne wird nur gering mobilisiert. Durch den Frakturverlauf wird in das Gelenk eingegangen und die Reposition kontrolliert.

Die Zugänge müssen in jedem Fall den bestehenden Weichteilläsionen und bei komplexen Frakturen am besten auch dem CT-Befund angepasst werden. Bei mindestens zweitgradig offenen Frakturen erfolgen ein temporärer Kunsthautersatz oder die Vakuumversiegelung und erst zweizeitig der Wundverschluss.

\section{Operationsmethode}

Grundlegende strategische Überlegung ist, dass die Kongruenz der Knöchelgabel von der Länge der Fibula und deren Einstellung in der Inzisur, dem intakten bzw. rekonstruierten Syndesmosenkomplex und stufenlosen Gelenkflächen abhängt.

\section{Operative Taktik}

- Exploration des OSG mit Entfernung kleiner Flakes oder ggf. Refixation größerer vitaler osteochondraler Fragmente

- Reluxation eingeschlagener Syndesmosen- bzw. Periost- oder Sehnenanteile

- Rekonstruktion der tibialen Gelenkfläche

- Fibulareposition in anatomischer Länge und Rotation

- Protektion der Syndesmosenheilung

- Innenknöchelversorgung

- Überprüfung der Lage und der Stabilität der Fibula und der freien OSGBeweglichkeit

\section{Operationsverfahren}

Die typischen Verfahren für die Versorgung der Außenknöchelbrüche können anhand der AO-Klassifikation aufgezeigt werden:

A-Frakturen. Sie werden nach direkter Reposition in Abhängigkeit von der Außenknöchelfragmentgröße mit transossärer Naht mit spät resorbierbarem Nahtmaterial (Stärke o oder kräftiger), mit einer Zuggurtungsosteosynthese (1,6-mm-/2,0mm-Kirschner-Draht mit 1,0-mm- bis 1,25-mm-Drahtcerclage) oder am besten mittels Zugschraubenosteosynthese mit 3,5-mm-Kortikalis- bzw. 4,o-mm-Spongiosaschraube versorgt.

Für die Indikation zur operativen Versorgung sind die Größe bzw. die Stellung des Fragments und v. a. die Instabilität entscheidend. Diese wird durch den Frakturverlauf, die mediale Frakturkomponente und den ggf. begleitenden ligamentären Schaden bestimmt. Maßgeblich wird das Ergebnis durch die Osteosynthese der medialen Adduktionsfraktur bestimmt. Daher werden bei A2- und A3Frakturen zunächst die mediale bzw.posteromediale tibiale bzw. malleolare Facette rekonstruiert und mit Zugschrauben stabilisiert. In seltenen Fällen ist hier auch eine mediale Abstützplatte bei proximaler Trümmerzone erforderlich (• Abb. 4).

B-Frakturen. Nach Reposition der Fibula und Sichern mit spitzer Repositions- oder Weller-Zange erfolgen die Zugschrauben-
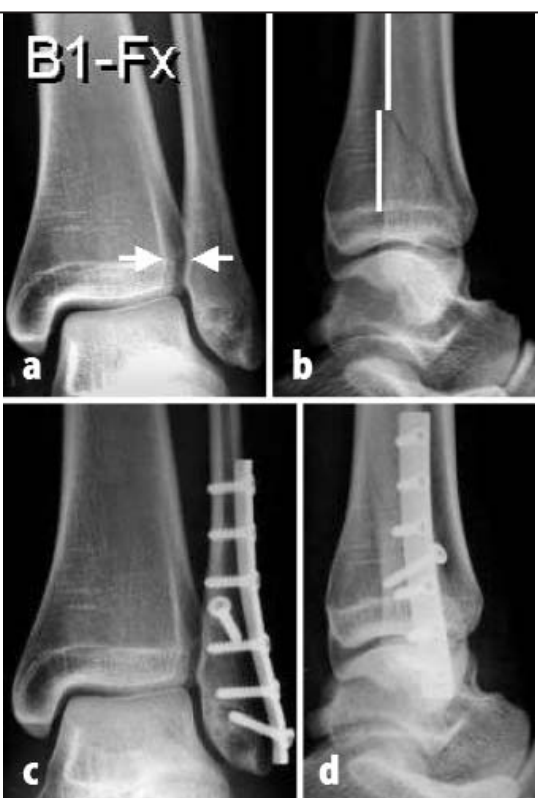

Abb. 5a-d $\Delta$ B1.2-Fraktur mit außenrotierter Fibula und geringer Dorsaldislokation (weiße Markierung) sowie erweitertem Syndesmosenspalt (weiße Pfeile; vermindertes tibiofibulares Overlapping,"TFO") $(a, b)$, postoperativ korrekte Einstellung $(c, d)$

osteosynthese von kranioventral nach dorsokaudal sowie die Anlage einer anmodellierten Drittelrohrneutralisationsplatte auf die Facies lateralis. Dabei sollte die Kortikalis proximal und distal mindestens 4-mal gefasst werden (• Abb. 5). Durch die korrekte Zugschraubenlänge können die Peronäalsehnen dorsal des Malleolus lateralis geschont werden. Um die fibulotalare Facette zu schonen, dürfen die distalen Plattenschrauben die Innenseite des Malleolus nicht perforieren. Alternativ kann, insbesondere bei kritischen lateralen Weichteilverhältnissen bzw. bei Versorgung über einen posterolateralen Zugang, dorsal eine (Antigleit-)Platte angebracht werden.

Bei den B2- und den B3-Frakturen erfolgen die Fibulaversorgung in oben genannter Weise und zusätzlich die Zugschraubenosteosynthese des Innenknöchels und der Volkmann-Komponente. Hier ist entsprechend der höhergradigen Instabilität häufig ein zweizeitiges Vorgehen erforderlich (• Abb. 6). Bei Mehrfragmentfibulafrakturen kann oft keine Zugschraubenosteosynthese erfolgen, sodass mit einer Platte überbrückend stabilisiert werden muss. 

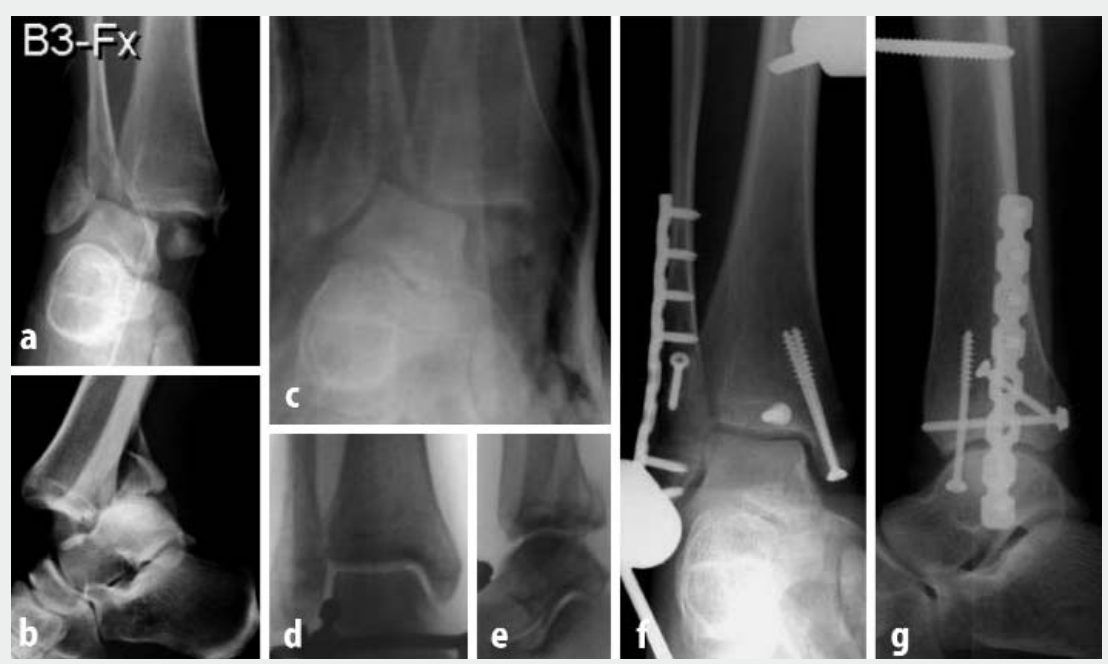

Abb. 6a-g $\Delta$ Hochgradig instabile B3-OSG-Luxationsfraktur (a,b), nach geschlossener Reposition im Gips nicht suffizient retinierbar (c), sofortige OSG-Transfixation (d, e); zweizeitige offene anatomische Reposition mit Platten- und Schraubenosteosynthese ( $f, g)$
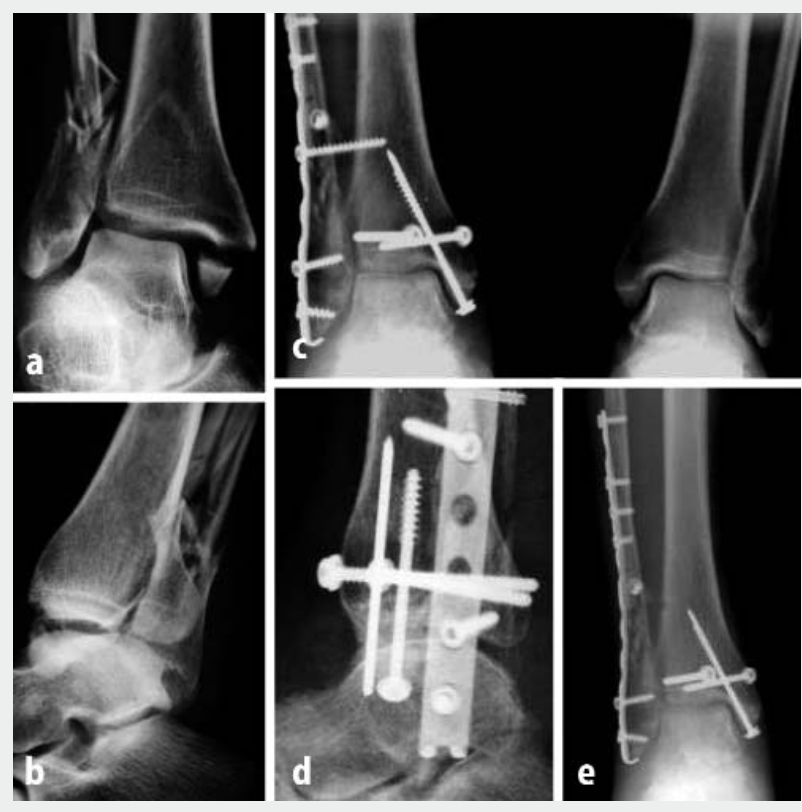

Abb. 7a-e $<$ C2.3-Luxationsfraktur $(a, b)$, aufgrund distaler Trümmerzone nur Versorgung mit überbrückender Plattenosteosynthese (zusätzlicher Stabilitätsgewinn durch das hakenförmig konfigurierte distale Plattenende) und "hoher" Stellschraube möglich; $(c, d)$ Belastungsaufnahmen im Seitenvergleich nach 6 Wochen im Stehen: anatomische Gelenkverhältnisse und korrekte Fibulaeinstellung vor und (e) nach Stellschraubenentfernung

C-Frakturen. Sie werden im diaphysären Anteil ebenfalls mit Platten- und Zugschraubenosteosynthese versorgt. Hier kommt wegen der größeren Primärstabilität häufig eine 3,5-mm-LC-DCP bzw.LCP zum Einsatz. Nach korrekter anatomischer proximaler Fibulareposition stellt sich der distale Anteil anatomisch in die Inzisur ein. Bei langstreckigen Frakturen sollte eine indirekte Reposition über eine proximal fixierte Platte erfolgen und distal nur das Hauptfragment im Sinne eine Überbrückungsosteosynthese an die Platte fixiert werden.
Bei Querfrakturen mit lokaler Trümmerzone kann die Anatomie des Fibulaquerschnitts als Repositionshilfe benutzt werden (palpable dorsolaterale Kante). Insbesondere bei allen komplexen (Segmentfrakturen/irreguläre Frakturen) Fibulafrakturen sollte die intraoperative Bildwandlerkontrolle des OSG mit Beurteilung eines stufenlosen Verlaufs der subchondralen tibiofibularen Gelenklinie im a.-p.-Bild genutzt werden (• Abb. 7).

Eine Besonderheit stellen hohe C-Frakturen (Maisonneuve-Frakturen) dar, deren Hauptproblem in der Verkürzung und der Rotationsfehlstellung liegt. Die stabilste Primärversorgung stellt auch hier die Plattenosteosynthese dar, welche zudem die beste distale Außenknöcheleinstellung garantiert, aber eine subtile Präparation unter Darstellung und schonender Retraktion des Peronäusnerven erfordert. Daher werden alternativ besser 2 Stellschrauben nach offener anatomischer Einpassung des Außenknöchels in die Inzisur eingebracht (s. unten). Für die Operationsindikation und zur Abgrenzung einer direkt traumatischen Fibulafraktur dient neben der Unfallanamnese bzw. klinischen OSG-Untersuchung die dynamische BVUntersuchung (• Abb. 8). Sie sollte auch zur Dokumentation der Verletzungsschwere für das berufsgenossenschaftliche Heilverfahren verwendet werden.

Verletzungen des Syndesmosenkomplexes. Es wird ein stufenweises Vorgehen entsprechend der Instabilität empfohlen. Beim lateralen Standardzugang kann die Syndesmosenregion von ventral dargestellt werden. Hierzu ist eine geringe Inzision der ventralen Faszie (distaler Anteil des oberen Extensorenretinaculums) erforderlich. Anschließend wird die Syndesmosenregion revidiert. Interponierte, freie knöcherne Fragmente werden entfernt und (osteo-)ligamentäre Anteile reponiert. Knöcherne Abrissfragmente als fibulares Wagstaffe-Fragment oder tibiales Tubercule de Chaput werden durch eine Minizugschraube evtl. mit U-Scheibe refixiert (๑Abb. 9). Die intraligamentär gerissene vordere Syndesmose wird fakultativ durch eine U-förmige Durchflechtungsnaht genäht, wobei diese lediglich der Adaptation der Bandstümpfe dient, oder besser durch eine transossäre Naht gesichert.

Die abschließende Stabilitätsprüfung nach der Osteosynthese (lateraler Hakenzug unter BV-Kontrolle, dorsale Schublade unter Daumendruck) entscheidet über die Notwendigkeit einer zusätzlichen Stellschraube. Die Stabilität wird weder durch den vorderen Syndesmosenanteil entscheidend determiniert noch durch deren Naht wiederhergestellt. Bei nachgewiesener relevanter Instabilität in oben genanntem Testverfahren ist von einer Ruptur der tiefen Anteile (Lig. tibiofibulare interosseum) auszugehen. In diesen 

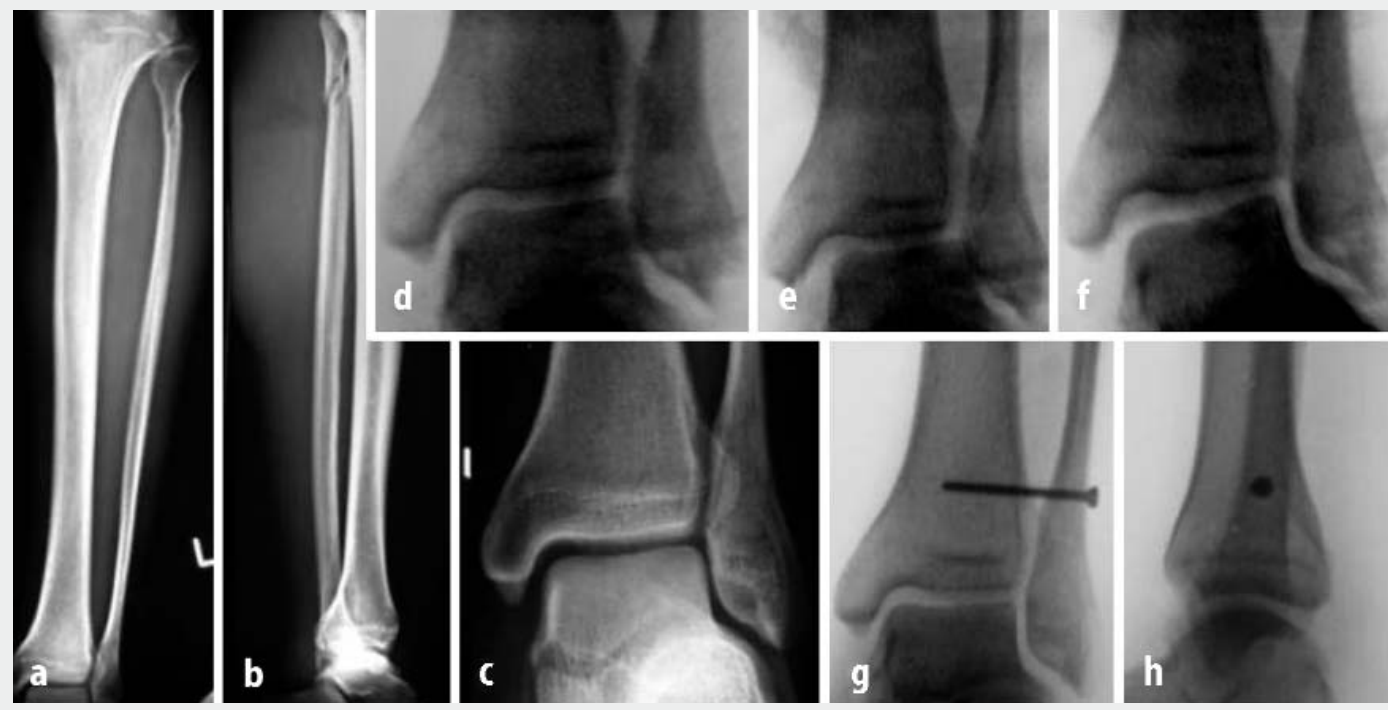

Abb. 8a-g $<$ C3-Maisonneuve-Fraktur $(a, b)$, OSGzentrierte unbelastete a.-p.-Röntgenaufnahme (c) bzw. BV-Einstellung (d), Lateralisationsstressuntersuchung mit erweitertem „medial clear space" (e),
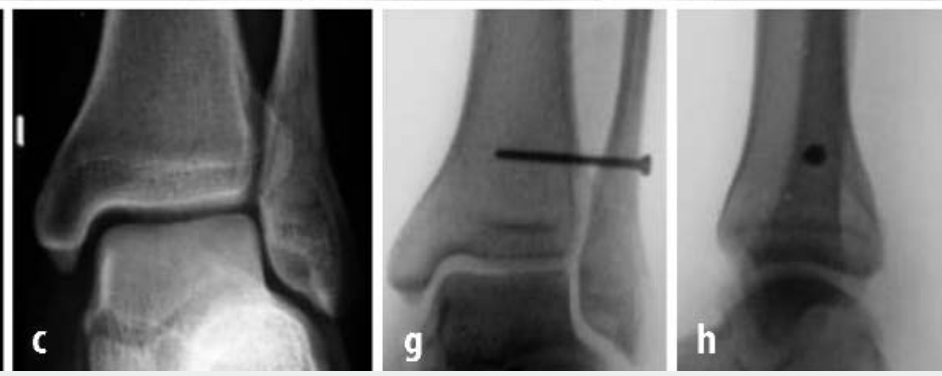

Valgusstress mit medialem „Tiltt" (f), korrekte Verhältnisse in der "mortise-view" nach Reposition und Stellschraubenimplantation (g), punktförmige Projektion der Schraube in der seitlich zentrierten Aufnahme mit sich überlappenden Malleolenspitzen (OSG-Achse) (h)
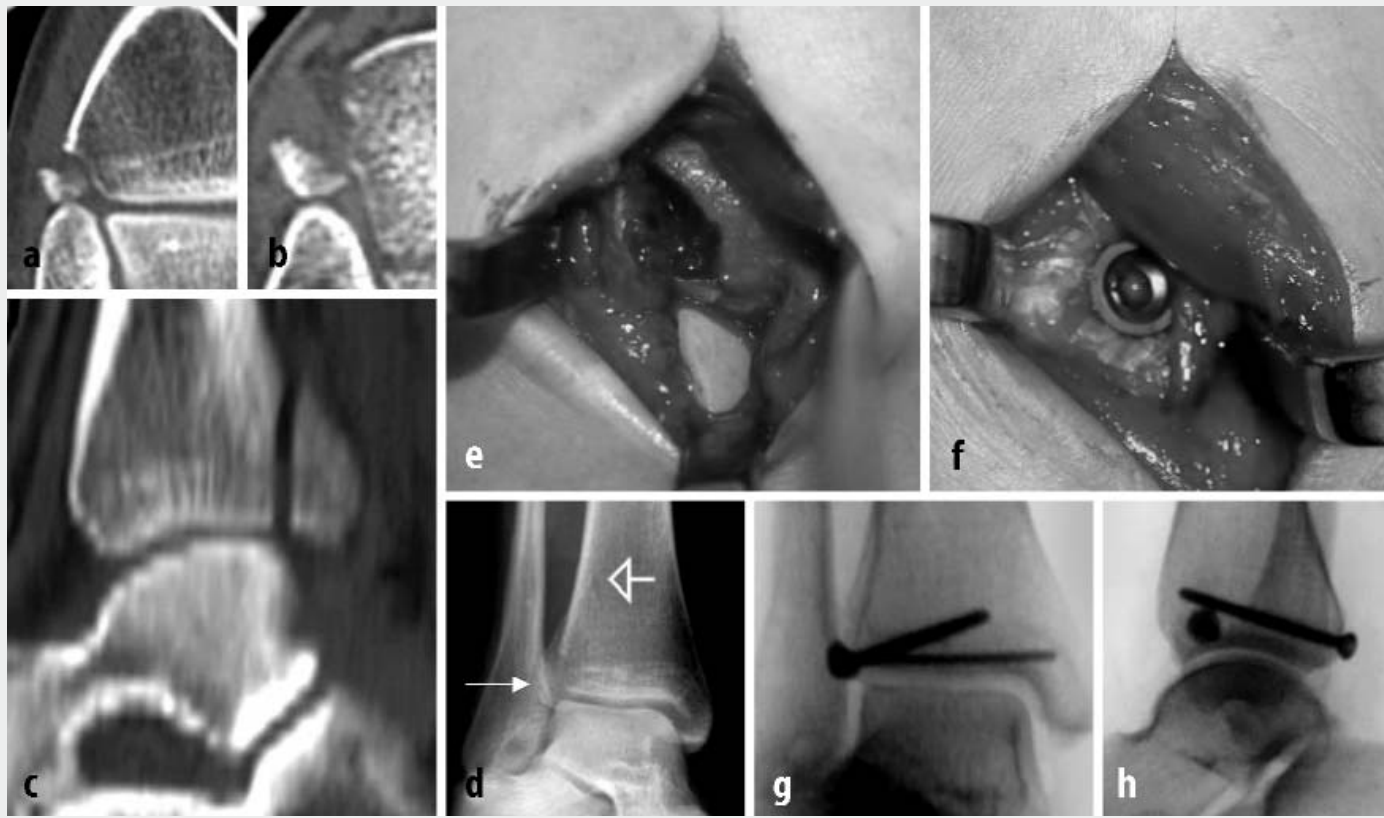

Abb. 9a-g $<$ Kombinierte knöcherne Abrissfraktur der vorderen und hinteren Syndesmose, in koronarer ( $a$, b) und sagittaler (c) CTSchicht gut darstellbar; in konventioneller Aufnahme sind beide Komponenten (Pfeile) nur bei genauer Betrachtung erkennbar (d), Operationssitus: Tubercule de Chaput vor (e) und nach (f) Verschraubung, BV-Aufnahmen: anatomische Stellung und korrekte Implantatlage $(g, h)$

Fällen ist die Indikation für eine temporäre Stellschraube gegeben.

Nach Einstellung der Fibula in die Inzisur und präliminarer Drahtfixation wird die Schraube nach Vorbohren und trikortikalem Gewindeanschnitt etwa 2$3 \mathrm{~cm}$ über dem OSG-Spalt eingebracht. Hierbei muss die Schraubenrichtung beachtet werden, die von lateral etwa $30^{\circ}$ nach ventromedial ansteigend verläuft (- Abb. 8). Bei kompletter intraligamentärer Ruptur aller Syndesmosenanteile und insbesondere bei additiver Deltabandläsion besteht eine hochgradige translatorische und rotatorische Instabilität. In diesen Fällen kann bei begleiten- der flacher Konfiguration der tibialen Facette des distalen Tibiofibulargelenks die korrekte Einstellung der Fibula problematisch sein. Um Fehlstellungen zu vermeiden, sollte in diesen Fällen die Fixation nach der Fibulaosteosynthese mit einer auf die Malleolenspitzen gesetzten Beckenrepositionszange erfolgen. Dadurch erfolgt die Reposition in der Achse des OSG. Diese Methode ist auch bei veralteten Revisionsfällen hilfreich.

Bei der sehr seltenen Luxation des Fibulaköpfchens wird analog über eine proximale Stellschraube fixiert.

Die Notwendigkeit zur Versorgung des bei etwa einem Drittel der Patienten vor- liegenden hinteren Kantenfragments besteht in Abhängigkeit von einer Gelenkflächenbeteiligung (Ausdehnung, Stufenbildung oder Impressionszone), bei bestehendem Repositionshindernis (Inzisur) oder bei persistierender Instabilität nach der Fibulastabilisierung. Röntgenmorphologisch kann zwischen den extraartikularen schalenförmigen Abrissfrakturen der hinteren Syndesmose (selten, <10\%; s. auch $\bullet$ Abb. 6 und 10), lippenförmigen Abscherfrakturen der tibialen Kante (etwa 50\%) und den eigentlichen Gelenkfragmenten (etwa 40\%; s. auch - Abb. 7) unterschieden werden. Für Letztere treffen auch die meisten der ein- 


\section{Sprunggelenkfrakturen}
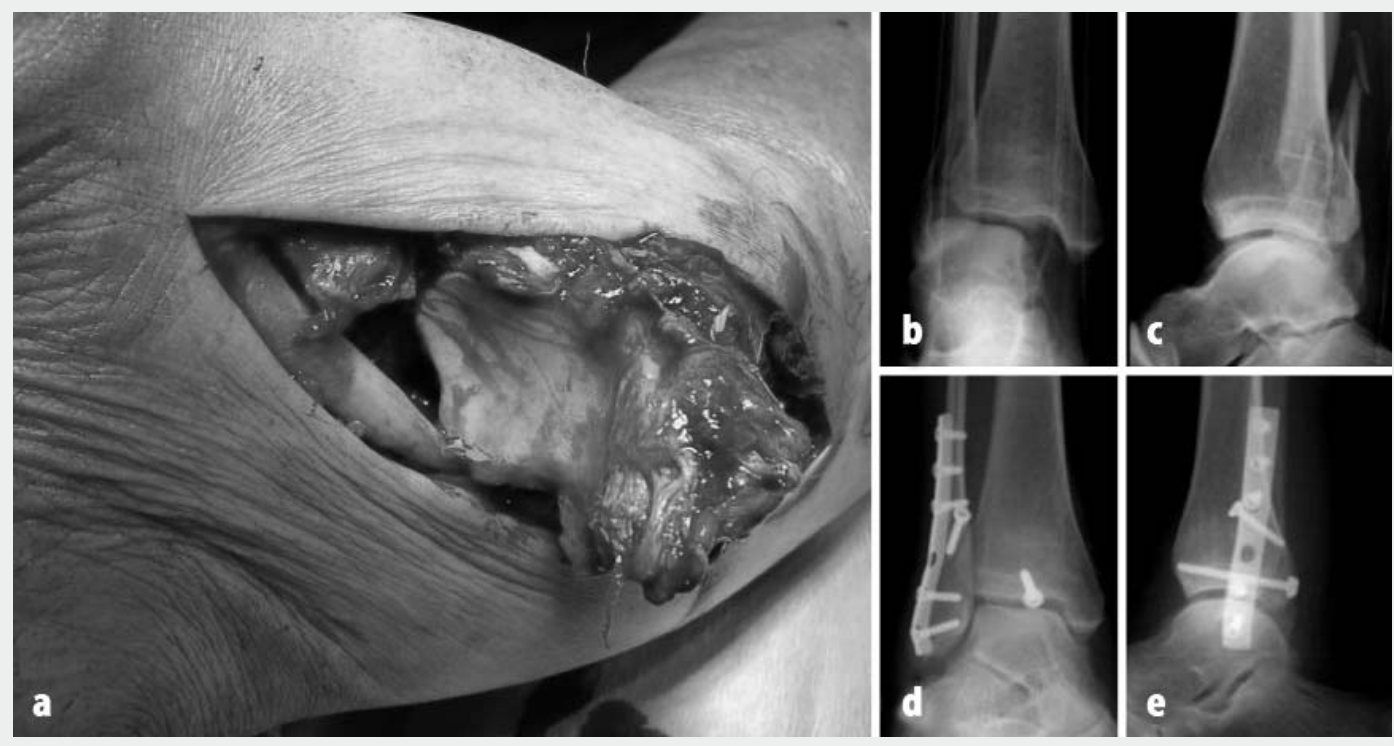

Abb. 10a-e $<$ B3-Fraktur mit kompletter Deltabandläsion und interponierter Postikussehne als Repositionshindernis, (a) notfallmäßige Versorgung über medialen Standardzugang, $(b, c)$ prä-, (d, e) postoperative Röntgenaufnahmen
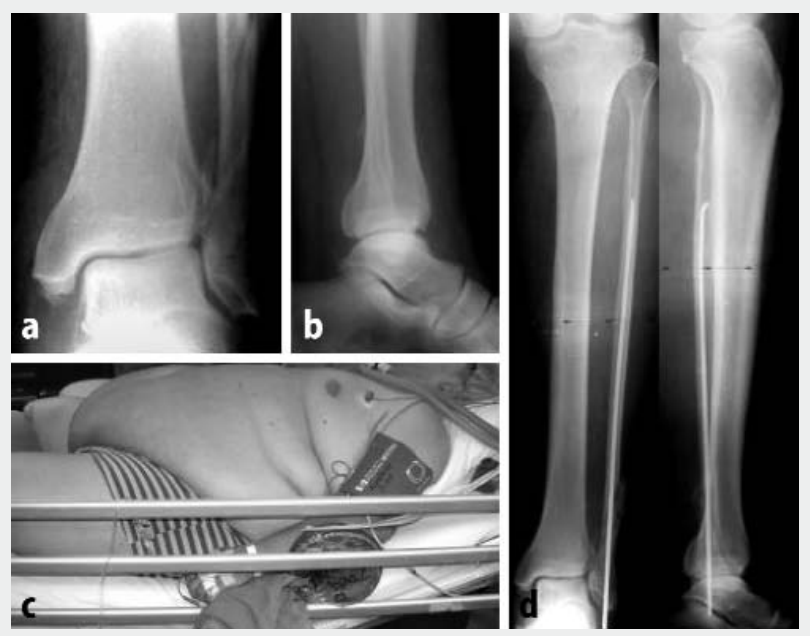

Abb. 11a-d $<$ Instabile C2-Verletzung mit knöchernem Deltabandabriss $(a, b)$ und schwerem Weichteilschaden mit lokaler Dermatose und systemischen Begleiterkrankungen (PAVK, Diabetes mellitus) (c), (d) perkutane TEN-Stabilisierung (titan elastic nail), Spätaufnahmen: Konsolidierung in korrekter Stellung

gangs erwähnten Operationsindikationen zu. Die offene Reposition unter Sicht zur Vermeidung von Inkongruenzarthrosen und stabile Fixation erfolgen am besten über den posterolateralen Zugang bei den schalenförmigen Fragmenten (cave: Verletzung der hinteren Syndesmose) bzw. über die posteromedialen Zugänge bei den Gelenkfragmenten. Bei größeren Impressionsbereichen kann über den medialen Standardzugang bei gleichzeitiger Innenknöchelfraktur durch eine schonende Reluxation des Talus nach posterolateral die gesamte tibiale Gelenkfläche gut eingesehen werden ( $\bullet$ Abb. 10). Daher sollte in diesen Fällen auch die Versorgung der tibialen Facetten vor der Außenknöchelstabilisierung durchgeführt werden.

In einigen Fällen kann auch durch die Fibulafraktur von lateral zwischen Tibia und Volkmann-Fragment eingegangen werden, und Repositionshindernisse können entfernt werden. Nachfolgend ist eine perkutane dorsale Verschraubung über eine posterolaterale Inzision möglich (- Abb. 9). Eine alleinige indirekte Reposition birgt auch unter Verwendung perkutaner Repositionshilfen die zusätzliche Gefahr des Verbleibens von Gelenkstufen und der Verletzung neurovaskulärer Strukturen.

Bei der Osteosynthese ist der direkten Verschraubung des Volkmann-Fragments von dorsal aufgrund der Stabilität der Vorzug zu geben. Falls eine indirekte perkutane Verschraubung von ventral durchgeführt wird, muss bei der anterioren Stichinzision auch auf eine sorgfältige Schonung des N. peronaeus superficialis geachtet werden, um schmerzhafte Neurome zu vermeiden. Weiterhin ist das Problem der Gewindelänge ohne Verzicht auf das Zugschraubenprinzip zu berücksichtigen.

Eine autologe Spongiosaplastik zur Unterfütterung angehobener Knorpelflächen kann lokal aus der distalen Tibia aus dem kranialen Anteil der Volkmann-Fraktur oder nach Schaffung eines kleinen Kortikalisdeckels gewonnen werden. Anschließend wird das Kantenfragment reponiert, durch temporäre Drahtfixation gesichert und mit möglichst 2 Kortikaliszugschrauben (evtl. mit U-Scheibe) definitiv versorgt. Nur bei Volkmann-Fragmenten, die mehr als ein Drittel der Gelenkfläche einnehmen, muss bei gleichzeitiger hochgradiger Osteoporose die Stabilisierung mit einer dorsalen Abstützplatte (z. B.T-Plättchen) erwogen werden. Anschließend wird bei Verwendung des 
posterolateralen Zuganges die Fibula mit einer dorsal angelegten Antigleitplatte und Zugschrauben durch die Platte stabilisiert. Der Vorteil liegt in der guten Weichteilbedeckung der Platte und der Fixierung des distalen Fragments durch Plattenschrauben ohne Gefahr der Gelenkperforation. Nachteilig kann die Plattenlage bei distalen Frakturen im Sulcus peronealis sein.

Extraartikuläre lippenartige Kantenfragmente oder nicht dislozierte schalenförmige Abrisse der hinteren Syndesmose bedürfen bei wiederhergestellter Stabilität keiner operativen Stabilisierung. Das Gegenstück stellt das anterolaterale Kantenfragment (Tubercule de Chaput) - die knöcherne Abrissfraktur der vorderen Syndesmose - dar (s. dort).

Innenknöchelfraktur. Sie wird nach epiperiostaler Darstellung unter Schonung der unmittelbar hinter dem Knöchel im Sulcus malleolaris verlaufenden Tibialis-posterior-Sehne durch Einzinkerzug retrahiert, vom Bruchhämatom gereinigt und nach Entfernung des eingeschlagenen Periosts direkt mit einem Kugelspieß reponiert. Anschließend wird temporär mit 2 parallelen 2,0-mm-Kirschner-Drähten fixiert, an der vorderen Gelenkkante durch die Kapselruptur das Repositionsergebnis klinisch und ggf. auch radiologisch kontrolliert und nachfolgend mit 2 4,0-mmSpongiosakurzgewindeschrauben definitiv stabilisiert. Diese können sukzessiv gegen die Kirschner-Drähte ausgetauscht und annähernd senkrecht zum Frakturverlauf ggf. mit U-Scheiben eingebracht werden. Bei kleineren Fragmenten wird neben einer Schraube ein Kirschner-Draht zur Rotationssicherung ( $\bullet$ Abb. 7) belassen bzw. selten eine reine Zuggurtungsosteosynthese bei knöchernem Bandausriss angewendet. Perkutane Schraubenosteosynthesen sollten nur bei unverschobenen Frakturen oder arthroskopisch assistiert durchgeführt werden. Bestehende größere ventromediale Impressionen werden zurückgestößelt, ggf. mit Spongiosatransfer unterfüttert und manchmal existierende osteochondrale Absplitterungen je nach Größe refixiert oder entfernt.
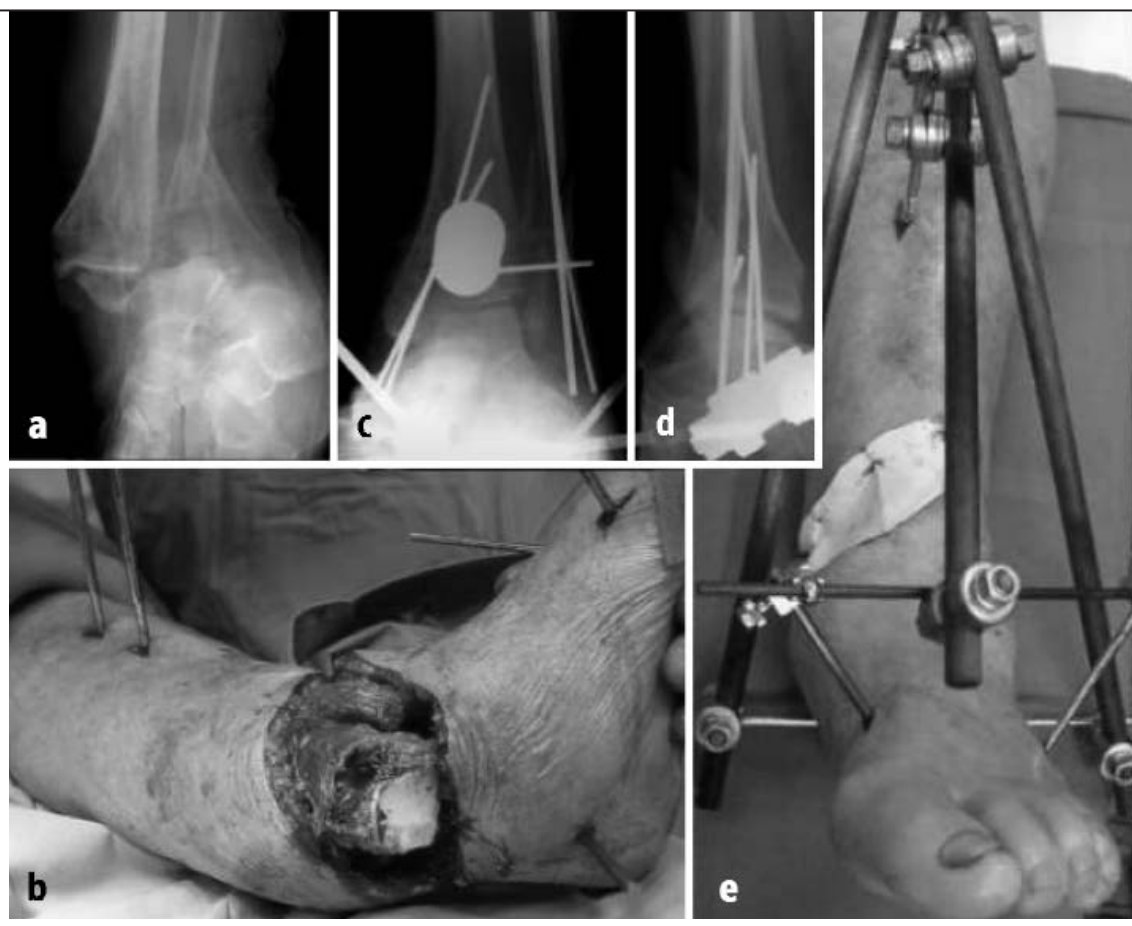

Abb. 12a-e $\Delta$ 3A-offene C2.2-Luxationsfraktur bei 96-jähriger Patientin mit 0steoporose (a), nach primärem Débridement und Jet-Lavage (b): minimalinvasive Drahtfixation des Innenknöchels, perkutane intramedulläre Schienung der Fibula und Kirschner-Draht-Transfixation des distalen Tibiofibulargelenks ( $c$, d) mit temporärem Hautersatz und Fixateur-externe-Ruhigstellung (e)

Deltabandrupturen. Sie sind meist partiell und brauchen in der Regel nicht gesondert versorgt werden. Eine Ausnahme stellt das sehr seltene mediale Repositionshindernis durch ein eingeschlagenes Deltaband, Periostanteile oder die Tibialis-posteriorSehne dar. Hier ist notfallmäßig eine offene Revision mit nachfolgender Adaptation oder - wenn möglich - transossärer Refixation der Deltabandanteile erforderlich (• Abb. 10).

Radiologische Kontrollen mit dem Bildwandler sind zur Überprüfung der erreichten Reposition, der Implantatlage bzw. der Stabilität nützlich und dienen der Dokumentation.

Weichteilschäden und Erkrankungen. Der Weichteilschaden, aber auch Allgemeinerkrankungen erfordern ein von dem aufgezeigten Algorithmus teilweise abweichendes Vorgehen, d. h. eine individuell abgestimmte Therapiestrategie. So muss bei vorliegendem Weichteilschaden mit lokalen und systemischen Begleiterkrankungen ggf. auf die Plattenosteosynthese verzichtet und nach der initialen Fixateurexterne-Ruhigstellung perkutan stabilisiert werden (• Abb. 11, 12).
Insbesondere ist in diesem Zusammenhang auf die steigende Inzidenz der (diabetisch) neuropathischen Osteoarthropathie (DNOAP) hinzuweisen. Obwohl die OSG-Beteiligung (Sanders-4-Lokalisation) bei der diabetischen PNP nicht die Hauptlokalisation darstellt, wird sie insbesondere auch bei Patienten nach Pankreas-Nieren-Transplantation vorgefunden. Hier muss neben der allgemein konservativen Vorgehensweise mit strikter Ruhigstellung und Langzeitentlastung für teilweise 4-6 Monate entsprechend der Akuität (Eichenholtz 1/2) die bestehende Immunsuppression beachtet werden (• Abb. 13). Diese „Frakturen“ sind als Erkrankung aus innerer Ursache aufzufassen. Daher muss eine genaue anamnestische Exploration erfolgen, und es dürfen entsprechende Bagatellursachen (Kausalitätsbedürfnis) nicht als wesentliche Ursache überwertet werden. Dementsprechend erfolgt die Behandlung regelhaft zu Lasten der GKV/PKV.

\section{Nachbehandlung}

Prinzip der postoperativen Therapie sind eine frühfunktionelle Nachbehandlung 

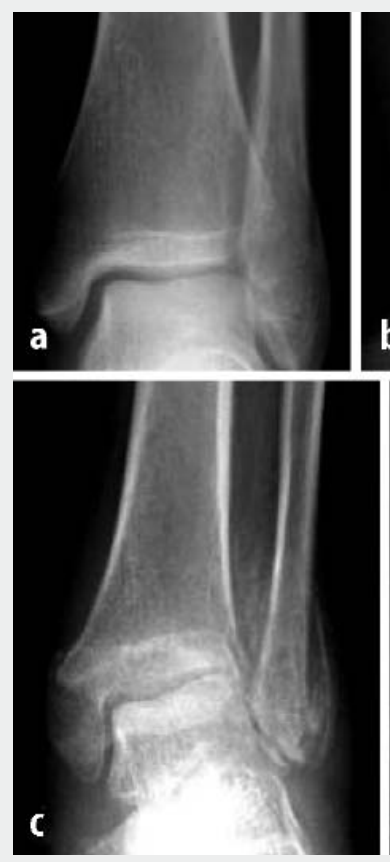
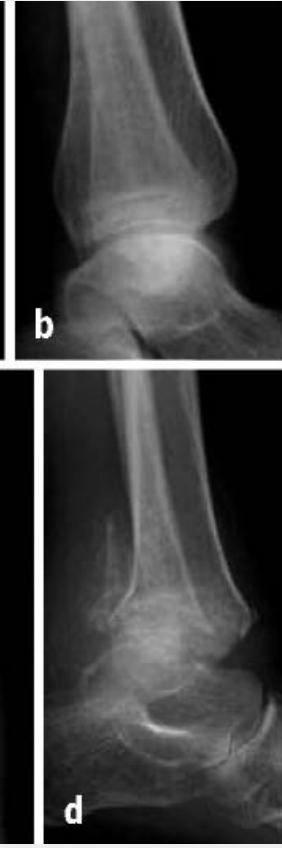

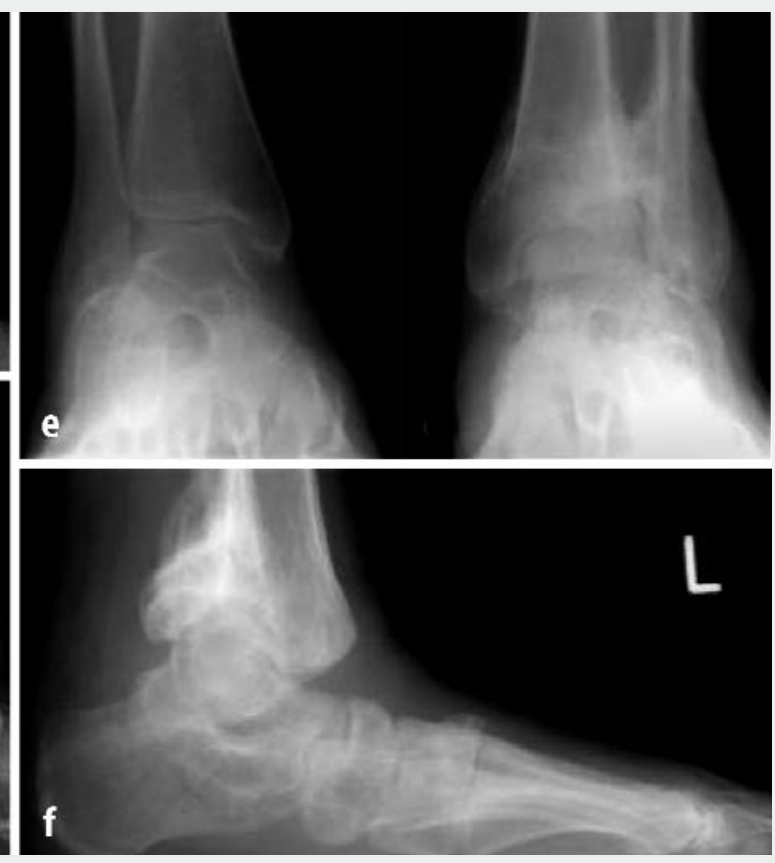

Abb. 13a-f $<$ Als nichtdislozierte stabile isolierte B1.1Außenknöchelfraktur $(a, b)$ eingeschätzte Sanders-4DNOAP im Stadium Eichenholtz 1 bei 29-jähriger $\mathrm{Pa}$ tientin nach PTx/NTx mit guter Organfunktion, aber noch persistierender PNP; nach 6 Wochen Castruhigstellung, aber ohne kompletter Entlastung: zunehmende Deformität (Eichenholtz 2) (c, d) und Konsolidierungsaufnahmen im Verlauf (Eichenholtz 3) $(\mathrm{e}, \mathrm{f})$ zur Optimierung der Gelenk- und Weichteiltrophik sowie der Propriozeption.

Die postoperative Ruhigstellung bis zur Wundheilung erfolgt ggf. mit einem Unterschenkelspaltgipsverband. Parallel dazu wird mit aktiven und passiven Bewegungsübungen aus dem Gips heraus sowie mit Lymphdränage begonnen. Nach der Entfernung der Redon-Dränagen kann der Patient mit 15-kp-Teilbelastung (gipsfrei) mobilisiert werden, falls eine gute Compliance besteht. Nur bei komplexen Rotationsinstabilitäten mit Nahtrefixation einer medialen kompletten Deltabandläsion muss eine 6 wöchige Castbehandlung erfolgen. Bei beidseitigen Frakturen oder Unmöglichkeit der einseitigen Entlastung kann eine entlastende Orthese, wie der Allgöwer-Gehapparat, zur Anwendung kommen. Bei isolierten (B1-)Außenknöchelfrakturen kann symptomadaptiert voll belastet werden.

Parallel dazu wird das krankengymnastische Übungsprogramm mit manueller, Elektro-, Kryo- und apparativ-isokinetischer Muskelfunktionstherapie durchgeführt, die auch eine Schulung der Propriozeption beinhaltet.

Nach spätestens 6 Wochen oder bei einem Wechsel des Therapiemodus sollte eine Röntgenkontrolle durchgeführt werden. Die Größe und der Schweregrad der Gelenkimpression entscheiden wesent- lich über die mögliche Belastungssteigerung.

Die Stellschrauben können über Stichinzisionen nach 6 Wochen in Lokalanästhesie entfernt werden, um dem physiologischen Spiel des Außenknöchels unter Vollbelastung und maximaler Dorsalextension Platz zu geben. Die Arbeitsfähigkeit sollte berufsabhängig meistens nach 3 Monaten erreicht sein.

Implantatentfernung. Die Indikation zur Implantatentfernung wird nach Ablauf etwa 1 Jahrs von lokalen direkten Beschwerden abhängig gemacht. Vorab sind aber eine sorgfältige Abklärung und Differenzialdiagnostik erforderlich, um posttraumatische Beschwerden durch artikulare oder Sehnenadhäsionen, ein Impingementsyndrom, eine Instabilität oder Arthrose usw. abzugrenzen. Die Implantatentfernung kann auch im Rahmen ggf. erforderlicher, funktionsverbessernder Eingriffe erfolgen. Reine Schraubenosteosynthesen, insbesondere von dorsal eingebrachte Volkmann-Fragmentschrauben, sollten aber in jedem Fall verbleiben.

\section{Komplikationen und Probleme}

Die häufigste Frühkomplikation ist das postoperative Hämatom, das großzügig revidiert werden sollte. Durch unfallbe- dingte Weichteilschädigung oder traumatisierende Operationstechnik bzw. schlechtes Timing sind Wundrandnekrosen möglich, die meist konservativ zur Abheilung gebracht werden können. Infektionen erfordern ein radikales chirurgisches mehrzeitiges Vorgehen mit ausgiebigem Débridement, Entfernung eines instabilen Implantats, ggf. externe Fixierung, evtl. mit plastischem Verschluss.

Pseudarthrosen sind nach operativer Therapie selten. Bei konservativer Primärbehandlung sind sie je nach Frakturtyp und Weichteilinterponat bzw. Repositionsergebnis beim Innenknöchelbruch in $30-60 \%$ zu erwarten und beim Außenknöchel meist mit einer Fehlstellung verbunden. Sie sollten nach radiologischer Diagnostik inklusive CT mittels Anfrischung und Kompressionsosteosynthese versorgt werden. Eine Spongiosaanlagerung ist selten erforderlich. Infektdefektpseudarthrosen werden nach den Prinzipien der septischen Chirurgie behandelt.

In Fehlstellung verheilte oder pseudarthrotisch konsolidierte Frakturen sind meist mit Fibulaverkürzung und Malrotation mit konsekutiver Taluslateralisation/-valgisation oder dorsaler Talussubluxation infolge fehlverheiltem VolkmannDreieck verbunden. Korrekturen müssen zeitlich limitiert angegangen werden, 
bevor arthrotische Veränderungen eingetreten sind. Sie sollten durch Korrekturosteotomie, Reosteosynthese, ggf. mit Interposition eines trikortikalen Beckenkammspans mit Syndesmosen- und Gelenkrevision behandelt werden. Eine lockere Malleolengabel bedeutet in der Regel eine in Fehlstellung (Verkürzung/ Außenrotation) verheilte Fibula, seltener eine rein ligamentäre Insuffizienz und wird wie oben beschrieben versorgt. Reine Syndesmoseninsuffizienzen können mit offener Revision des distalen Tibiofibulargelenks und Bandersatzplastik mittels Periostlappenplastik oder Sehneninterponat versorgt werden.

Bei Gelenkkontrakturen, Impingementsyndromen, lokalen Knorpelschäden oder freien Gelenkkörpern kann eine arthroskopische Therapie mit arthroskopischem Offset, Adhäsiolyse, ggf. Microfracturing usw. erfolgen.

Zur Therapie der häufig erst spät auftretenden posttraumatischen symptomatischen Arthrose steht die Möglichkeit einer Endoprothesenimplantation zur Verfügung, falls keine persistierende chronische Osteitis vorliegt. Die modernen 3-Komponenten-OSG-Prothesen werden als ungeführte Prothese mit tibialer und talarer zementfreier Verankerung mit einem mobilen PE-Gleitkern implantiert. Hierbei muss ggf. über eine vorab notwendige oder einzeitig-simultan durchzuführende Korrekturosteotomie entschieden werden. Falls keine Alloarthroplastik möglich oder gewünscht ist, kann eine OSG-Arthrodese durchgeführt werden. Diese erfolgt meist als Schraubenarthrodese nach Entknorpelung, Synovektomie, ggf. Spongiosaauffüllung und Einbringung von 3 tibiotalaren Zugschrauben, ggf. einer weiteren fibulotalaren Schraube in plantigrader Neutralstellung des Fußes. Alternativ kann bei geringgradigen Fehlstellungen eine arthroskopische Fusion mit perkutaner Schraubenarthrodese bzw. bei Infektdefektsituation, bei kritischen Weichteilverhältnissen und osteoporotischen Knochen die Kompression mit einem Fixateur externe, am besten mit der Ilisarov-Composite-Hybridtechnik, durchgeführt werden.

\section{Prognose}

Konservativ behandelte Luxationsfrakturen weisen in großen Sammelstatistiken nur in etwa der Hälfte der Fälle gute Ergebnisse auf, während übungsstabil versorgte und frühfunktionell nachbehandelte Brüche in etwa $80 \%$ ein einwandfreies Resultat zeigen. Hierbei besteht eine Korrelation zur Frakturschwere von der A-Fraktur mit 95\% bis zur C-Fraktur mit etwa $75 \%$ guten und sehr guten Endergebnissen. Eine Früharthrose ist meist auf eine unvollständige Reposition bzw. Retention des Volkmann-Fragments, fehlende anatomische Einstellung der Fibula in der Inzisur oder auf den traumatischen Knorpelschaden zurückzuführen.

\section{Fazit für die Praxis}

Für ein optimales Ergebnis ist ein abgestuftes Therapieschema erforderlich, das den Weichteilschaden und den Frakturtyp bzw. Schweregrad der Instabilität, aber auch bestehende Erkrankungen in die Behandlungstaktik einbezieht.

Entsprechend den differenten Ausgangsbedingungen werden die Therapieoptionen verschieden sein. Aber nur wenn der strategische Grundsatz der anatomischen Gelenkflächenrekonstruktion und Wiederherstellung der korrekten Position der Gelenkpartner zueinander als Ziel bestehen bleibt, werden langfristig gute Ergebnisse erzielt werden können.

\section{Korrespondierender Autor \\ Dr. D.-H. Boack}

Sektion Fuß- \& Sprunggelenkchirurgie, Klinik für Unfall- und Wiederherstellungschirurgie, Charité Campus Virchow,

Augustenburger Platz 1, 13353 Berlin

E-mail:dan-henrik.boack@charite.de

Interessenkonflikt: Der korrespondierende Autor versichert, dass keine Verbindungen mit einer Firma, deren Produkt in dem Artikel genannt ist, oder einer Firma, die ein Konkurrenzprodukt vertreibt, bestehen.

\section{Literatur}

1. Chapmann MW (1993) Fractures and fracture-dislocations of the ankle. In: Mann RA, Coughlin JC (eds) Surgery of the foot and ankle, 6th edn, vol 2. Mosby, St Louis, pp 1439-1464
2. Hahn DM, Colton CL (2000) Malleolar fractures. In: Rüedi HP, Murphy WM (eds) A0 principles of fracture management.Thieme, Stuttgart New York, pp 559-582

3. Hintermann B (1998) Arthroskopie bei Frakturen des oberen Sprunggelenkes. Arthroskopie 11: 252-258

4. Richter J, Schulze W, Muhr G (1999) Stabile Knöchelbrüche - Indikation zur Operation oder konservativen Therapie. Orthopäde 28: 493-499

5. Zwipp H (1994) Chirurgie des Fußes. Springer, Berlin Heidelberg New York, S 161-167 